IRRITABLE BOWEL SYNDROME

\title{
Brain functional magnetic resonance imaging of rectal pain and activation of endogenous inhibitory mechanisms in irritable bowel syndrome patient subgroups and healthy controls
}

\author{
C H Wilder-Smith, D Schindler, K Lovblad, S M Redmond, A Nirkko
}

Gut 2004;53:1595-1601. doi: 10.1136/gut.2003.028514

See end of article for authors' affiliations

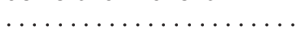

Correspondence to:

Dr C H Wilder-Smith,

Brain-Gut Research

Group, Bubenbergplatz

11, CH-3011 Berne,

Switzerland; cws@ggp.ch

Revised version received 13 February 2004

Accepted for publication 30 March 2004

\begin{abstract}
Background and aims: Many patients with irritable bowel syndrome (IBS) show intestinal hypersensitivity to distension and sensitisation after repeated intestinal distensions. Abnormalities in endogenous pain inhibitory mechanisms, such as diffuse noxious inhibitory controls (DNIC), may be implicated and were investigated during brain functional magnetic resonance imaging (FMRI).

Patients and methods: FMRI was performed in 10 female patients with IBS (five constipated (IBS-C) and five with diarrhoea (IBS-D)) and 10 female healthy controls during rectal balloon distension alone or during activation of DNIC by painful heterotopic stimulation of the foot with ice water. Rectal pain was scored with and without heterotopic stimulation $(0=$ none, $10=$ maximal $)$.

Results: Heterotopic stimulation decreased median rectal pain scores significantly in healthy controls $(-1.5$ (interquartile range -2 to -1$) ; p=0.001)$ but not in IBS-C $(-0.7(-1$ to 0.5$))$, IBS-D $(-0.5(-1.5$ to 0.5$)$ ), or in all IBS patients $(0(-1.5$ to 1.3$))$. Brain activation changes during heterotopic stimulation differed highly significantly between IBS-C, IBS-D, and controls. The main centres affected were the amygdala, anterior cingulate cortex, hippocampus, insula, periaqueductal gray, and prefrontal cortex, which form part of the matrix controlling emotional, autonomic, and descending modulatory responses to pain.

Conclusions: IBS-C and IBS-D appear to have differing abnormal endogenous pain inhibitory mechanisms, involving DNIC and other supraspinal modulatory pathways.
\end{abstract}

I rritable bowel syndrome (IBS) is characterised by abdominal discomfort or pain accompanied by changes in gastrointestinal motility. ${ }^{1}$ The underlying mechanisms remain unclear but visceral hypersensitivity is apparent in many patients with IBS. Rectal distension studies have shown up to $60 \%$ of IBS patients have lower sensory thresholds compared with healthy controls, depending on the selection of patients and the distension protocol. ${ }^{1-9}$ Differences in pain scores or somatic referral areas during distension are inconclusive when comparing IBS patients and controls. ${ }^{71}{ }^{11}$ Sensitisation to repeated intestinal distension has been considered specific for IBS, as healthy subjects did not develop significant rectal sensitisation of discomfort ratings after sigmoid conditioning. ${ }^{8}$ These data imply altered sensory processing in IBS, possibly involving central and spinal sensory modulation. ${ }^{812}{ }^{13}$ Major endogenous pain modulating mechanisms include the periaqueductal grey (PAG)-rostroventral medulla (RVM) network and the spinobulbo-spinal diffuse noxious inhibitory controls (DNIC). ${ }^{14} 15$ They are central in regulating pain perception and have been shown to be abnormal in several conditions associated with IBS, such as fibromyalgia. ${ }^{16}{ }^{17}$ Assessment of DNIC using the counterirritation technique is well validated and widespread in pain research. DNIC activation is quantified by the perceptual modulation of a painful stimulus by a secondary heterotopically applied nociceptive, generally thermal or electrical stimulus. ${ }^{14-22}$

Hypersensitivity may be secondary to peripheral or central changes in the nervous systems. Peripheral sensitisation with subsequent spinal and supraspinal sensitisation provides a suitable mechanism for lowered sensory thresholds. The minimal inflammatory changes demonstrated in subgroups of IBS patients may represent an adequate stimulus. ${ }^{23}$ Hypervigilance, increased selective attention to potentially threatening stimuli, is a putative central component of sensitisation leading to overrating of stimulation intensities. Brain imaging techniques have demonstrated abnormal central processing of rectal pain in areas dealing with affective and emotional sensory processing in IBS. ${ }^{16}{ }^{24-26}$ These areas are intricately involved in the integration of autonomic responses and in supraspinal afferent input modulation. No published data exist on imaging of brain function during activation of endogenous antinociception and DNIC during visceral pain and, although malfunctioning modulation has been repeatedly postulated, little is know about the functional intactness of endogenous pain modulation in IBS.

The aim of this study was to examine DNIC in IBS subgroups and healthy controls during functional magnetic resonance imaging (fMRI), by modulation of visceral pain with heterotopic nociceptive stimulation.

\section{METHODS}

Twenty right handed females, aged 20-50 years, were recruited for this trial. Ten patients with IBS (Rome 2 criteria $^{27}$ ) as their main complaint and a weekly average pain score greater than $30 \mathrm{~mm}$ on a $100 \mathrm{~mm}$ horizontal visual

\footnotetext{
Abbreviations: $\mathrm{ACC}$, anterior cingulate cortex; $\mathrm{BOLD}$, blood oxygen level dependent; DNIC, diffuse noxious inhibitory controls; fMRI, functional magnetic resonance imaging; IBS, irritable bowel syndrome; IBS-C, IBS with constipation; IBS-D, IBS with diarrhoea; PAG, periaqueductal gray; PET, positron emission tomography; RVM, rostroventromedial medulla; VOI, volumes of interest; VAS, visual analogue scale
} 
analogue scale (VAS: $0=$ none, $100=$ unbearable) during a one week run-in period were recruited from our outpatient clinic. Five patients were constipated (IBS-C: $<1$ hard motion every third day, strong defecatory straining) and five had diarrhoea (IBS-D: $>3$ soft or liquid motions per day). The healthy control group consisted of 10 females recruited by word of mouth, with no gastrointestinal pathology or history of abdominal pain, bloating, discomfort, constipation, or diarrhoea more than twice a week in the last three months. Exclusion criteria in all groups were clinically relevant gastrointestinal, hepatic, or other systemic disease, lactose intolerance, bowel resections (except appendectomy) or abdominal operations, any medication, treatment with any investigational drug during the preceding 30 days, pregnancy or lactation, and inability to give written informed consent. The University of Berne ethics committee approved the study.

Whole brain fMRI scans were acquired during rectal distension with and without heterotopic somatic stimulation for assessment of brain activation due to DNIC. Before imaging, a lubricated latex balloon with virtually infinite compliance up to a distension volume of $800 \mathrm{ml}$ and attached to the end of a catheter was introduced into the rectum with subjects in the left lateral position, inflated to $200 \mathrm{ml}$, and gently pulled outwards until slight resistance was felt. The balloon was tested for leakage before insertion and the catheter attached to a hand held syringe. Rectal distension thresholds for first sensation (first slight fullness), defecatory urge ("begin to look for toilet"), pain detection (first feeling of pain), and pain tolerance ("would deflate balloon because of strong pain") were titrated twice with subjects in a comfortable supine position using a manually controlled continuous ramped inflation rate of $25 \mathrm{ml} / \mathrm{s}$. Pain detection threshold volumes from the second determination plus $20 \%$ were used during fMRI. Subsequently, the heterotopic cold pressor test was performed by immersing the left foot in an ice water bath maintained at $4{ }^{\circ} \mathrm{C}$ and pain intensity rated after 120 seconds on a $100 \mathrm{~mm}$ anchored horizontal visual analogue scale. This test was subsequently used for somatic heterotopic stimulation during rectal distensions.

After a resting period, subjects were positioned inside the MRI scanner, head motion was minimalised by use of a head frame and a bite plate, and subjects kept their eyes closed. Ear plugs were used to protect against gradient noise. MR imaging was performed on a commercial whole body $1.5 \mathrm{~T}$ system equipped with a circular polarised head coil (Siemens Magnetom Vision, Erlangen, Germany). A preliminary three plane scout image was acquired before initiation of the echo planar fMRI sequences for whole-brain $\mathrm{T}^{*}$ imaging ( $\mathrm{Tr}$ 6 seconds; TE $84 \mathrm{~ms}$; flip angle $90^{\circ}$ ). A total of 30 slices with a 128 pixel matrix, $5 \mathrm{~mm}$ thickness, $4 \mathrm{~mm}$ slice distance between slice centres (1 mm overlap, interleaved acquisition), and 68 measurements were performed per experimental run. Each run was performed twice with each subject, resulting in a total of 136 whole brain measurements per subject. There was a break of 10 minutes between runs to ensure rewarming of the ice water cooled foot. Slices were placed parallel to a line connecting the genu corporis callosi and confluens sinuum, in order to minimise echo planar induced susceptibility artefacts and to cover the whole brain with a minimum of slices. ${ }^{28}$ To minimise effects due to anticipation of pain, most likely at the transients between rest and painful stimuli, two sample volumes (12 seconds) were excluded from analysis at each time point of change (balloon inflation/pressure release).

Eight rectal distensions of 48 seconds duration each were performed, separated by 48 second rest periods with complete balloon deflation. Heterotopic stimulation was performed during the third, fourth, seventh, and eighth distensions and extended into the immediately following baseline rest periods. The resulting $2 \times 2$ design allowed assessment and comparison of activation due to balloon distension versus rest both during and without heterotopic stimulation. To avoid motion artefacts due to leg movements, the ice water was filled into and then drained from a water bath positioned before the start of fMRI. Subjects reported rectal pain ratings (verbal rating scale: $0=$ none, $10=$ unbearable) after each pair of distensions (1st and 2nd, 3rd and 4th, 5th and 6th, and 7th and 8th).

A structural high resolution spin-echo-train inversion recovery magnitude sequence was then run to acquire anatomical data for localisation and a high resolution three dimensional MPRAGE sequence for future co-registration. Images were exported to a Sun workstation (Sun Microsystems, Palo Alto, USA) for post-processing. Both quantitative volume of interest (VOI) analysis and visualisation with colour coded $\mathrm{z}$ score maps were performed using purpose written software. ${ }^{29}$ For quantitative assessment of brain activation in predefined cortical areas with respect to interindividual variability of anatomical details, we used a modified anatomically defined parcellation scheme, as described by Rademacher et al, and subsequently adapted for fMRI. ${ }^{28-31}$ Due to the high resolution, each subject's own echo planar imaging data were suitable for both blood oxygen level dependent (BOLD) fMRI and for structural anatomical definition of VOIs. The following VOIs were defined and individually delineated: paracentral gyri (sensorimotor cortex Sl and Ml), mesiotemporal region (amygdala, hippocampus), anterior cingulum, posterior cingulum, anterior insula, posterior insula, PAG, supramarginal gyrus (secondary sensory cortex S-II), orbitofrontal cortex, inferior dorsolateral prefrontal cortex (along inferior frontal sulcus), superior dorsolateral prefrontal cortex (along superior frontal sulcus), anterior, posterior, medial, and lateral quadrants of the thalamus, and the occipital visual cortex (control area). VOIs were assessed separately in each hemisphere. The time series was analysed for each VOI (see below).

Independent of VOI analysis, voxel based z score images were generated for visualisation according to standardised procedures. $^{30}$ Cortical activation in response to balloon distension was separately evaluated in both cases for the phases during and without heterotopic stimulation. Activation was defined as a BOLD signal increase relative to baseline and deactivation as a BOLD signal decrease. One time sample point of each VOI consisted of the average signal intensity of all pixels in that VOI at the specific whole brain measurement at that time, resulting in a time series of 136 measurements per VOI. Excluding two time samples (12 seconds) at each transition to allow for inflation or deflation of the balloon as well as for the cerebral haemodynamic response, the means and variances of each of the four phases (with and without balloon distension, each with and without heterotopic stimulation) were determined for each VOI, allowing statistical comparisons with the parametric z test for normal distribution of large samples. Z score limits for a given $\mathrm{p}$ value were Bonferroni corrected for the number of VOIs measured. Because VOIs were individually delineated and anatomical regions corresponded, VOI data could be reliably pooled for each group.

Per cent BOLD signal intensity changes due to balloon inflation were compared with the respective baseline and calculated separately for phases without and with heterotopic stimulation. Furthermore, the difference between this balloon inflation induced activation (per cent change) during heterotopic stimulation, minus the analogue balloon inflation induced activation (per cent change) without heterotopic stimulation, was calculated as the effect of the heterotopic modulation on balloon induced activation related to each VOI. This is possible when comparing per cent signal changes but 
Table 1 Rectal balloon distension thresholds and pain/discomfort during tonic rectal distension in 10 controls and 10 irritable bowel syndrome

\begin{tabular}{|c|c|c|c|c|c|c|c|}
\hline & $\begin{array}{l}\text { lst sensation } \\
\text { (ml) }\end{array}$ & $\begin{array}{l}\text { Defecation urge } \\
\text { (ml) }\end{array}$ & $\begin{array}{l}\text { Pain detection } \\
\text { (ml) }\end{array}$ & $\begin{array}{l}\text { Pain tolerance } \\
\text { (ml) }\end{array}$ & $\begin{array}{l}\text { Tonic distension } \\
\text { during fMRI (ml) }\end{array}$ & $\begin{array}{l}\text { Distension: pain } \\
\text { (VAS 0-100) }\end{array}$ & $\begin{array}{l}\text { Distension: discomfort } \\
\text { (VAS 0-100) }\end{array}$ \\
\hline Controls & $41(24-58)$ & $118(93-143)$ & $195(142-248)$ & $270(204-335)$ & $227(170-284)$ & $52(36-68)$ & $48(32-64)$ \\
\hline IBS-all & $53(24-81)$ & 157 (118-195) & $271(194-347)$ & $363(263-463)$ & $320(226-414)$ & $47(38-56)$ & $43(28-57)$ \\
\hline IBS-C & $63(14-100)$ & $183(101-265)$ & $322(153-491)$ * & $443(239-647)^{*}$ & $386(183-590)+$ & $47(30-65)$ & $47(20-74)$ \\
\hline IBS-D & $42(21-63)$ & 130 (103-157) & $219(184-254)$ & $283(235-331)$ & $253(211-295)$ & $47(31-63)$ & $38(15-61)$ \\
\hline
\end{tabular}

Means (95\% confidence intervals) are shown.

IBS-C, five patients with IBS with constipation; IBS-D, five patients with IBS with diarrhoea; VAS, visual analogue scale $(0=$ none, $100=$ unbearable); fMRI, functional magnetic resonance imaging.

${ }^{*} \mathrm{p}<0.05, \mathrm{IBS}-\mathrm{C}$ versus healthy controls and IBS-D; $\uparrow \mathrm{p}=0.03$, IBS-C versus healthy controls.

not when using z scores (for extensive statistical discussion see Nirkko and colleagues ${ }^{29}$ and Jaeggi and colleagues ${ }^{31}$ ).

$\mathrm{Z}$ test for large samples was used to assess statistical significance of the signal changes with respect to baseline, between conditions (using appropriately combined variances) and between groups. The $\mathrm{z}$ score limits for a given $\mathrm{p}$ value were based on two tailed analysis, and Bonferroni correction was made for the number of VOIs measured in the VOI analysis. Likewise, for voxel based visualisation, clusters were included with at least one voxel with a z score above 5.0, which corresponds to a full Bonferroni correction for a $\mathrm{p}<0.05$ for all voxels of the complete brain. Additionally, the extent of such clusters was displayed with different colours coded down to a $\mathrm{z}$ score of 2.0, which correspond to an uncorrected $\mathrm{p}<0.05$.

\section{Statistical analysis of group data}

All continuous group data are shown as means (95\% confidence intervals (CI)). Analysis of variance (ANOVA) testing was used for comparisons within and between groups. Tukey's honest significance difference test was used for confirmative testing. Non-parametric data were expressed as medians and analysed using the Kruskal-Wallis and Mann-Whitney $U$ tests. A significance level of $p<0.05$ was applied throughout.

\section{RESULTS}

\section{Subject characteristics}

Mean ages (95\% CI) did not differ significantly between healthy controls, IBS-C, and IBS-D: 31 years (24-38), 35 years (25-45), and 40 years (24-57), respectively. Mean weight and height in the respective groups were $61 \mathrm{~kg}$ (55$66)$ and $168 \mathrm{~cm}(164-171), 58 \mathrm{~kg}(53-63)$ and $166 \mathrm{~cm}$ (161$171)$, and $61 \mathrm{~kg}(50-72)$ and $167 \mathrm{~cm}$ (155-179). IBS had been present for $2-5$ years in two patients and for over five years in three patients in both IBS groups.

\section{Rectal distension threshold}

Rectal distension threshold balloon volumes were significantly different between groups (ANOVA group effect $\mathrm{p}=$ 0.03) (table 1). Pain detection and tolerance volumes were significantly higher in IBS-C than in healthy controls or IBS$\mathrm{D}(\mathrm{p}<0.05)$. Distension resulted in similar pain and discomfort ratings after 120 seconds in all subject groups (table 1).

Distension pain ratings without and with heterotopic stimulation of the first and second series were pooled as there were no significant differences between the two series. Rectal pain ratings without and with heterotopic stimulation were significantly different in healthy subjects $(p=0.001)$ but not in IBS-C or IBS-D. The decrease in rectal pain scores with heterotopic stimulation was significantly greater in healthy subjects than in IBS-C and IBS-D $(p<0.01)$ (fig l). Mean pain scores during the cold pressor test alone were not significantly different between subject groups.

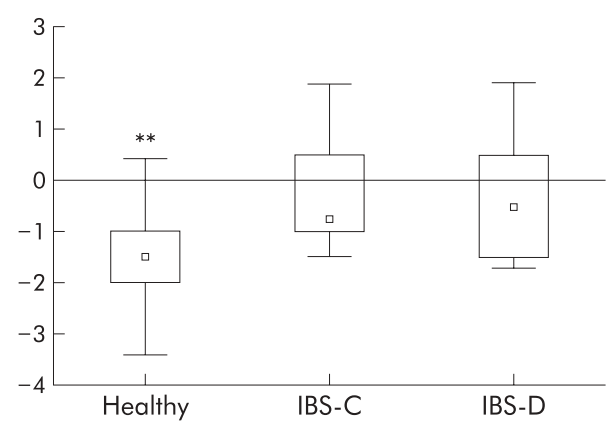

Figure 1 Differences in rectal distension pain ratings $10=$ no pain, $10=$ unbearable pain) with and without painful heterotopic ice water immersion of the foot in 10 healthy subjects and five constipated (IBS-C) and five diarrhoeic (IBS-D) irritable bowel syndrome (IBS) patients. Negative values denote decreased rectal pain with heterotopic stimulation. Medians, interquartile ranges, and total ranges of repeated two distensions are shown. ${ }^{* *} p<0.01$, healthy subjects versus IBS-C and IBS-D.

\section{Brain $\mathrm{fMRI}$}

There were significant differences in activation and deactivation in specific VOIs between healthy controls and IBS patients during painful rectal distension without and with heterotopic stimulation (tables 2, 3; figs 2, 3). In healthy controls, areas of activation and deactivation were generally bilateral whereas asymmetry was common in the IBS subgroups (data summarised in tables 2 and 3). Activation patterns during rectal pain in IBS-C and IBS-D were different to healthy controls but also from each other (table 2). Notably, the anterior perigenual cingulate, most of the prefrontal and SI cortices, and the posterior insula were significantly activated during rectal pain in controls but not in either IBS group compared with baseline. IBS-D showed markedly increased activation of the thalamic nuclei and lack of activation of most other VOIs compared with baseline and with controls and IBS-C.

Activation responses during heterotopic stimulation are shown in table 3 and again demonstrate major differences between healthy and IBS subjects. IBS-D differed from IBS-C substantially during heterotopic stimulation by only showing significant deactivation in a single VOI, the anterior right insula. Increased activations of the amygdala and hippocampus were unique to IBS-C, as was deactivation of the PAG in healthy controls. No significant activation changes were seen in the control area, the visual cortex, in any group of subjects.

Examples of averaged fMRI time courses for the right inferior prefrontal VOI, right anterior insula, and left visual cortex (control region) in healthy controls and the IBS groups are shown in fig 4.

\section{DISCUSSION}

Differences in activation of endogenous noxious inhibitory pathways were clearly demonstrated between healthy controls and subgroups of IBS patients using a validated 
Table 2 Brain regions significantly activated during rectal pain compared with baseline ( $z$ score $>3$, Bonferroni corrected, two tailed)

\begin{tabular}{|c|c|c|c|}
\hline & Controls & IBS-C & IBS-D \\
\hline $\begin{array}{l}\text { Cingulate anterior } \\
\text { Cingulate posterior } \\
\text { Prefrontal cortex inferior } \\
\text { Prefrontal cortex superior } \\
\text { Insula anterior } \\
\text { Insula posterior } \\
\text { Thalamus lateral } \\
\text { Thalamus medial } \\
\text { Thalamus anterior } \\
\text { S1 } \\
\text { SII } \\
\text { Orbitofrontal } \\
\text { Amygdala/hippocampus }\end{array}$ & $\begin{array}{l}\text { +Bilateral* } \\
\text { +Bilateral } \\
\text { +Bilateral } \\
\text { +Bilateral } \\
\text { +Bilateral } \\
\text { +Bilateral } \\
\text { +Bilateral } \\
\text { +Bilateral } \\
\text { +Bilateral } \\
\text { +Bilateral } \\
\text { - Right }\end{array}$ & $\begin{array}{l}\text { +Left } \\
+ \text { Leftt } \\
\text { +Right } \\
\text { +Left } \\
\text { +Bilateral } \\
\text {-Bilateral } \\
\text {-Bilateral§ }\end{array}$ & $\begin{array}{l}\text { +Bilateral } \\
\text { +Right } \\
\text { +Bilateral } \\
\text { +Right } \\
\text { +Right } \\
\text { - Left }\end{array}$ \\
\hline
\end{tabular}

method of counterirritation during rectal distension pain. This was evident in clinical pain measures as well as in brain activation by fMRI. Additionally, previously reported differences in brain activation during visceral pain between IBS patients and controls were corroborated and extended. $.^{16} 24-2632$ Marked group differences in pain processing between IBS patients with diarrhoea and constipation were shown for the first time.

Noxious input underlies multiple endogenous regulatory mechanisms at the spinal and supraspinal levels, which can be both facilitatory and inhibitory. Major modulation of pain perception is coordinated in the PAG-RVM network, with extensive input from the limbic forebrain, diencephalon and brainstem, and the spino-bulbo-spinal DNIC pathway. ${ }^{33}$ DNIC is a potent source of endogenous analgesia, which has, for example, been shown to be abnormal in fibromyalgia. ${ }^{17}{ }^{18}$ Dysfunction in modulatory pathways has also been hypothesised in IBS but has yet to be demonstrated. ${ }^{813} 16$ Assessment of DNIC using the counterirritation technique is well validated and widespread in pain research. DNIC activation is quantified by perceptual modulation of a painful stimulus by a secondary heterotopically applied nociceptive stimulus and has been shown to be distinct from distraction. $^{14}{ }^{15}$ 17-22 $3435^{35}$ DNIC is thought to rely on spino-bulbospinal loops with minimal input from the PAG-RVM or other supraspinal pathways. ${ }^{15}{ }^{36}{ }^{37}$ In the current study, heterotopic ice water stimulation predictably decreased rectal pain intensity in healthy controls, indicating effective activation of inhibitory pathways. Concurrent changes in cerebral blood flow in the forebrain, diencephalon, and limbic areas were demonstrated, indicating coactivation of supraspinal, possibly PAG-RVM, pathways. Major changes in activation during heterotopic stimulation were seen in the anterior cingulate cortex (ACC, Brodman 24' and 32'), the insular cortex, and the prefrontal cortex, which have extensive connections to the PAG and RVM. ${ }^{15}{ }^{38}$ Diminished activation during heterotopic stimulation in healthy controls in the anterior and posterior insula, the medial thalamus, and the PAG may reflect promotion of inhibitory feedback loops. PAG lies at a crossroads for neural circuits that coordinate rapid and profound somatic, autonomic, and antinociceptive modulation..$^{39}$ The observed effects are not due to the cold pressor test itself, as all values were baseline corrected.

The absence of a significant inhibitory effect on visceral pain in either IBS subgroup suggests impaired activation of inhibitory controls, and brain activation patterns differed markedly from healthy controls, but also between subgroups. During heterotopic stimulation only IBS-C demonstrated significant activation of the amygdala, hippocampus, and thalamus, and also in the cingulate (Brodman 32') and prefrontal cortices.

Table 3 Brain regions significantly activated by heterotopic stimulation during rectal pain (z score $>3$, Bonferroni-corrected, two tailed)

\begin{tabular}{|c|c|c|c|}
\hline & Controls & IBS-C & IBS-D \\
\hline $\begin{array}{l}\text { Cingulate anterior } \\
\text { Cingulate posterior } \\
\text { Prefrontal cortex inferior } \\
\text { Prefrontal cortex superior } \\
\text { Insula anterior } \\
\text { Insula posterior } \\
\text { Thalamus lateral } \\
\text { Thalamus medial } \\
\text { Thalamus anterior } \\
\text { S1 } \\
\text { PAG } \\
\text { Orbitofrontal } \\
\text { Amygdala/hippocampus }\end{array}$ & $\begin{array}{l}\text { +Bilateral* } \\
\text { +Bilateral } \\
\text { +Bilateral† } \\
\text { +Bilateral } \\
\text { - Bilateralf } \\
\text { - Left } \\
\text {-Right } \\
\text { +Bilateral } \\
\text { - Midline }\end{array}$ & $\begin{array}{l}\text { +Rightt* } \\
\text { +Bilateral† } \\
\text { +Bilateral } \\
\\
\text { +Bilateralई } \\
\text { +Bilateral } \\
\text { +Bilateral }\end{array}$ & -Right \\
\hline
\end{tabular}

IBS, irritable bowel syndrome; IBS-C, IBS with constipation; IBS-D, IBS with diarrhoea; PAG, periaqueductal gray +, Significant activation; - , significant deactivation; Bilateral, bilateral symmetrical activation changes; Right or Left, respective asymmetrical activation changes.

*Increased versus IBS-D; tincreased versus IBS-D; $\ddagger$ decreased versus IBS-C and IBS-D; sincreased versus healthy and IBS-D; $\bullet$ increased versus healthy; ${ }^{*}$ increased versus healthy and IBS-D; $+\dagger \S \S$ increased versus healthy and IBS-D. 

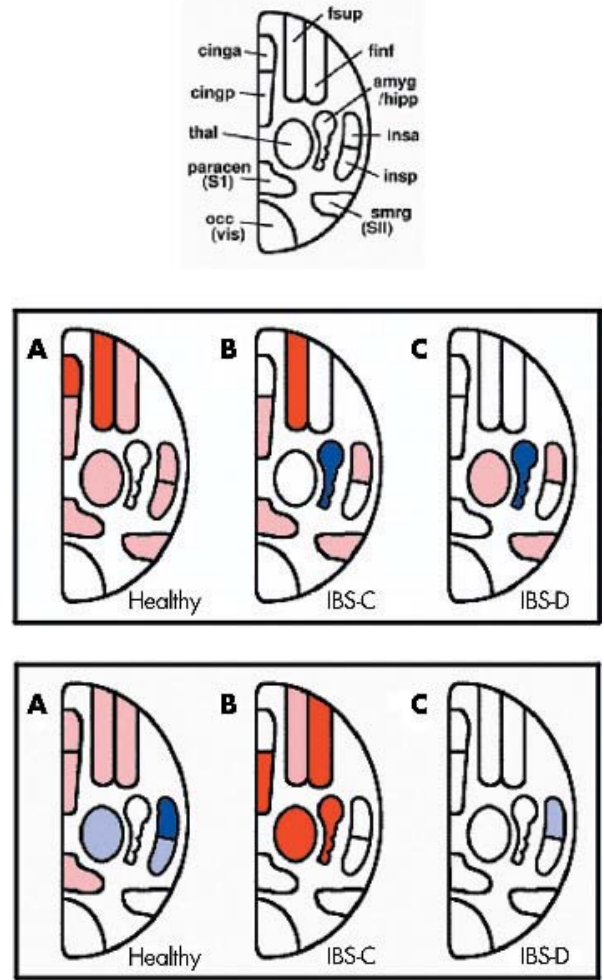

Figure 2 Schematic representation of the main volumes of interest (VOI) defined for the functional magnetic resonance imaging analysis (top). Abbreviations: fsup, superior dorsolateral prefrontal cortex; finf, inferior dorsolateral pretrontal cortex; amyg/hipp, amygdala and hippocampus; insa, anterior insula; insp, posterior insula; smrg, secondary sensory cortex; occ, occipital visual cortex; paracen, primary sensory cortex; thal, thalamus; cingp, posterior cingulate cortex; cinga, anterior cingulate cortex. Significantly increased or decreased activations during painful rectal distension without (middle row) or with (bottom row) painful heterotopic stimulation of the foot compared with baseline are shown in healthy controls, and constipated (IBS-C) and diarrhoeic (IBS-D) irritable bowel syndrome (IBS) patients. VOls significantly activated or deactivated compared with baseline within each group are shaded in pink and light blue, respectively. Significant activations or deactivations compared with other subject groups are depicted in red or dark blue, respectively. For detailed results of all activated VOls, please refer to table 2.

IBS-D differed profoundly from IBS-C and controls in only showing decreased activation in the right anterior insula during heterotopic stimulation. The main divergent activations in the IBS subgroups were seen in the brain regions integrating emotions, attention, and cognition, implying dysfunction in the corresponding modulation of nociceptive input. It has been previously proposed by Villanueva et al, that DNIC may play a physiological role in the detection of nociceptive signals and also constitute both a filter, which allows extraction of the signal for pain, and an amplifier in the transmission system, which increases the potential alarm function of the nociceptive signals. ${ }^{36}$ Malfunction of this filter would explain several of the postulated sensory disorders in IBS.

Evidence for dysfunction in rectal pain processing without counterirritation has previously been presented in mixed groups of IBS patients. ${ }^{162-26324041}$ This study corroborates earlier data and adds additional information by separately considering IBS subgroups. In controls, significant bilateral activation compared with baseline was seen during painful rectal stimulation in most of the brain centres classically associated with pain perception and processing, such as the cingulate, insular, prefrontal cortices, the SI and SII regions,
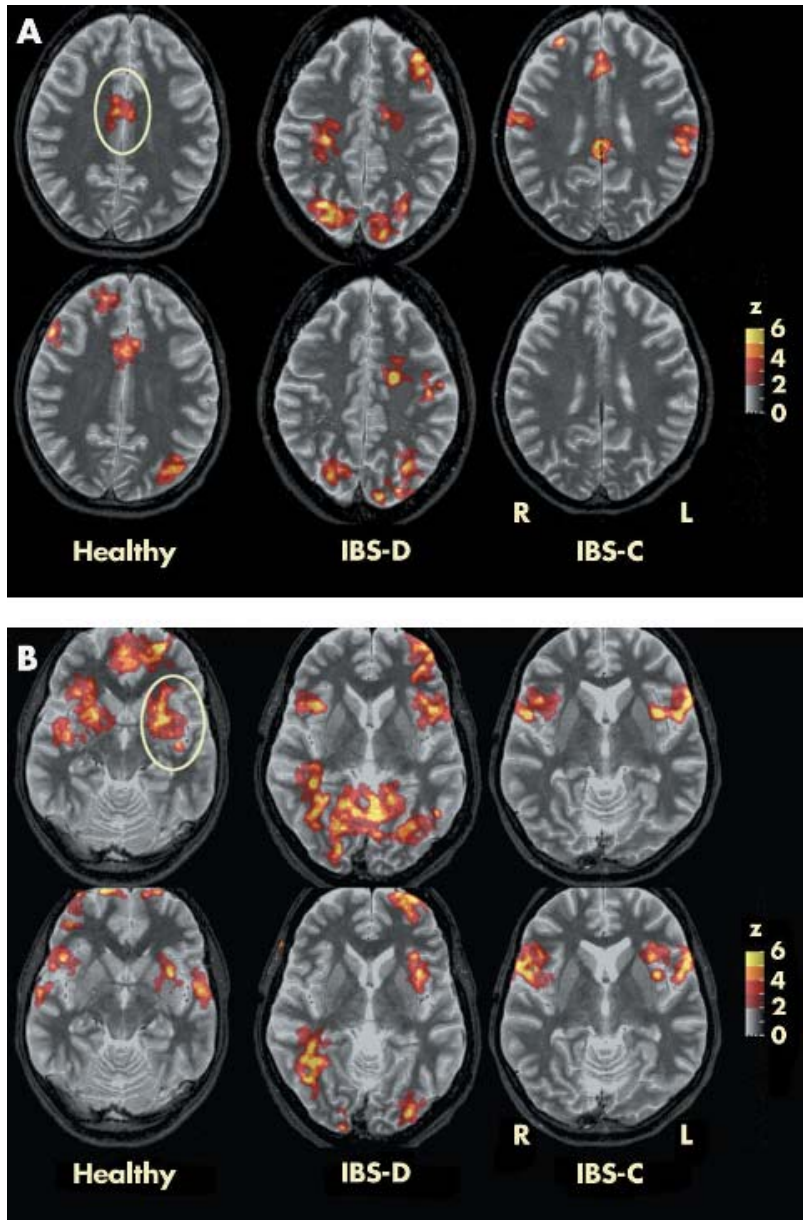

Figure 3 Functional magnetic resonance imaging in a representative healthy control, and a constipated (IBS-C) and diarrhoeic (IBS-D) irritable bowel syndrome (IBS) patient during painful rectal distension without (top row) and with painful heterotopic stimulation (bottom row) at sections through the anterior cingulate (A) and insula (B). The anterior cingulate and insula cortices are encircled. Clusters with significant differences from baseline are depicted as colour coded values (see $\mathrm{z}$ scale bar).

the thalamus, and limbic system. ${ }^{42-49}$ The regions activated are constituents of a complex matrix of connections involved in the primary sensory analysis and secondary integration of emotional, affective, memory, autonomic, and motor responses, as well as feedback and feed-forward regulation of nociceptive stimulation. ${ }^{40} 4550$ Activation patterns in the IBS subgroups differed from each other as well as from controls, except for the sensory discriminative SI and SII pain centres, where patterns were similar between all groups, excluding the clear hemispheric lateralisation in both IBS groups. In contrast with healthy controls, both IBS groups showed no significant bilateral activation in the anterior, perigenual cingulate, and prefrontal cortices during painful distension compared with baseline. The absence of ACC activation during rectal pain in IBS patients probably demonstrates a resetting of the gain of the secondary integrative pain processing system and a pre-existing saturation in the entire pain/anxiety network. An analogous seemingly paradoxical absence of cortical activation due to baseline saturation has been observed in other conditions, such as the Gilles de la Tourette syndrome. ${ }^{51}$ Decreased activation of the ACC has been previously demonstrated in IBS patients where it was interpreted as a diminished antinociceptive response to aversive visceral events, and also 

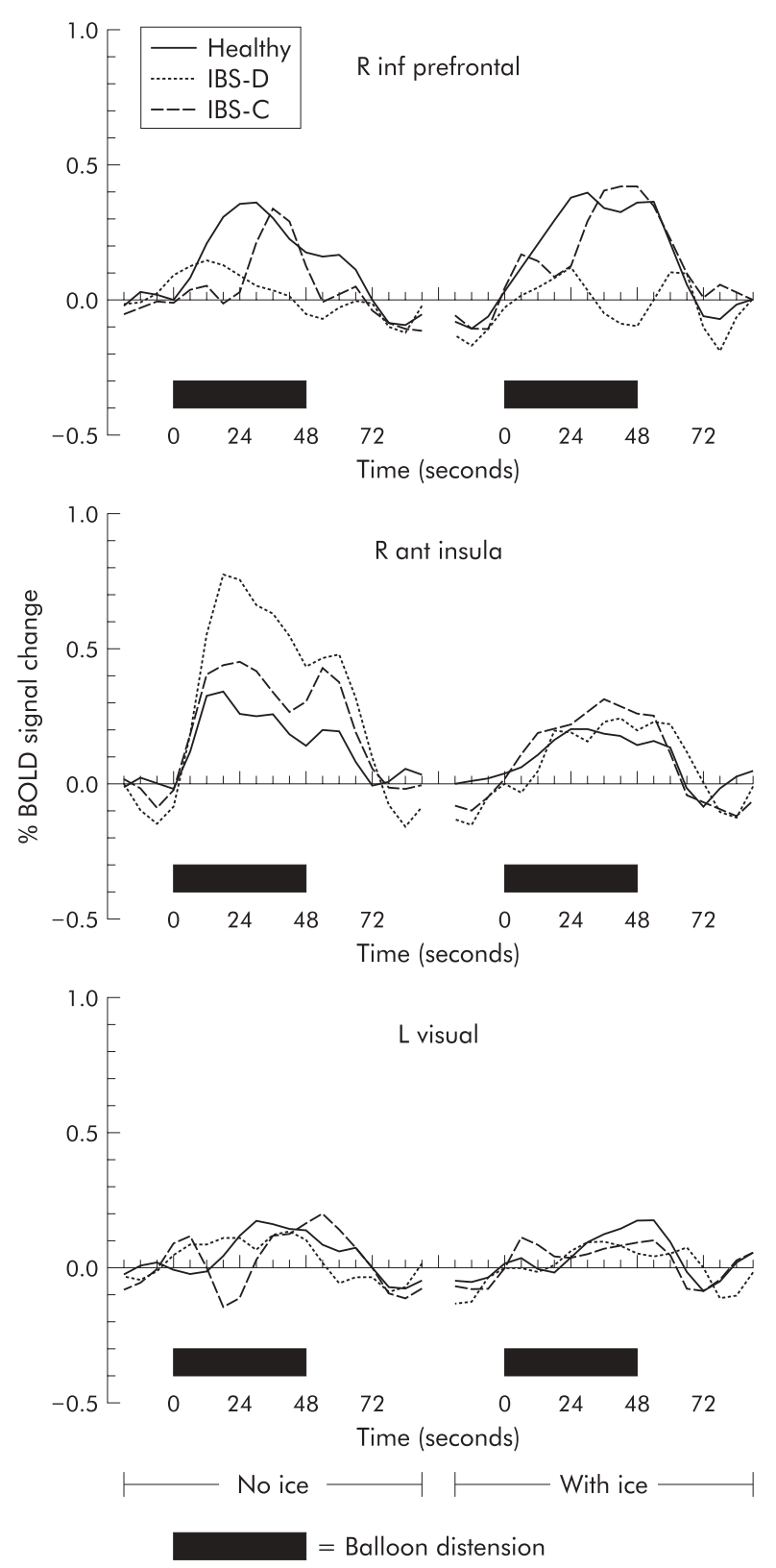

Figure 4 Averaged functional magnetic resonance imaging time courses (BOLD signal change) for right inferior prefrontal ( $R$ inf prefrontal) volumes of interest, right anterior insula ( $R$ ant insula), and the left visual cortex (L visual, control region) in healthy controls, and constipated (IBS-C) and diarrhoeic (IBS-D) irritable bowel syndrome (IBS) patients. Black bars mark balloon inflation. Responses to rectal balloon inflation without (left graph) and with (right graph) heterotopic stimulation are shown. Note delay of BOLD responses due to both haemodynamic delay and time needed to inflate/deflate the balloon.

in depressed patients. ${ }^{24} 5253$ However, in mixed groups of IBS patients, trends to increased activation of the ACC have also been described, emphasising the importance of patient, as well as stimulus selection. ${ }^{25} 2641$ In the current study, all patients had significant visceral pain during the study and pain stimulation was individually titrated to similar levels, explaining some discrepancies in imaging data to previous studies. The ACC is a central player in the neural network governing fear and anxiety, otherwise comprised of the amygdala, hippocampus, prefrontal cortex, insular cortex, ventral striatum, and PAG, and also modulates control of arousal and attention and of motor reactions and response selection. ${ }^{54-58}$ It appears to be critical for integrating chronic pain with memory, allowing full appreciation and evaluation of the meaningfulness of the stimulus in light of previous experience and for integration with autonomic responses and control of hypothalamic stress responses. ${ }^{44} 45$

Both IBS groups showed deactivation during pain and activation with heterotopic stimulation in the amygdala, and in the hippocampus, which initiates behavioural, autonomic, and endocrine responses to noxious stimuli. ${ }^{26558}$ This effect was significant in IBS-C and was not observed in healthy controls. Dysfunction in IBS patients in the hypothalamicpituitary axis is clinically evident in abnormal stress responses. ${ }^{59}$ Pain related anxiety normally leads to hippocampal activation and correlates with activity in the perigenual ACC and midinsular regions. ${ }^{60}{ }^{61}$ Considerable group differences were seen in thalamic activation. IBS-C showed no increased activation in any thalamic region while IBS-D had increased activity in more nuclei than healthy controls. The majority of nociceptive information entering the brain from the spinal cord is received by nuclei in the thalamus. The anterior thalamic nuclei are closely linked to behavioural, spatial, and memory functions, while the ventral posterior lateral regions and the nucleus gracilis play an important role in the transmission of visceral sensory information and in viscerosomatic convergence and modulation, which are modulated by attentional circuitry. ${ }^{62-64}$ The intralaminar and medial nuclear complexes are involved mainly in the mediation of long term information about the state of the viscera and of emotional components of viscerosensory impact, including pain. ${ }^{36}$ Both lateral and medial thalamic nuclei possess neurones encoding pain intensity and most functional imaging studies of acute pain in healthy subjects have demonstrated significant activation in these regions. ${ }^{40626365}$ Thalamic activation differences from controls imply aberrant sensory encoding or feedback regulation from cortical and limbic centres in IBS.

Potential weaknesses of this study include, firstly, the small patient numbers due to splitting of the IBS patients into subgroups. However, pooling of the data was deemed inappropriate due to the marked differences between the groups. Highly significant and consistent effects were demonstrated but minor effects may have been insignificant due to inadequate statistical power. The current data need to be confirmed in larger groups of patients. Secondly, manual volume based distensions rather than barostat pressure controlled inflations were used due to difficulties with the use of a barostat in the MRI scanner room. This is unlikely to affect the results as titrations to individual perception levels were performed using a rapid phasic inflation paradigm. Pain threshold volumes were significantly higher in IBS-C than in IBS-D, as has previously been demonstrated for pressure thresholds. ${ }^{66}$ However, small numbers and the relative insensitivity of volume versus pressure thresholds preclude further speculation. Thirdly, because of the coexistence of inhibitory and excitatory neurones in many of the involved centres, a causal correlation between visualised activations and function is currently not possible using fMRI techniques.

In summary, activation of inhibitory noxious controls by counterirritation in healthy controls activated extensive supraspinal pathways, probably including the PAG-RVM pathways. Constipated and diarrhoeic IBS patients differed significantly from each other and healthy controls in their inappropriate brain activation responses to rectal pain without and with counterirritation. The centres affected were part of the matrix controlling emotional, autonomic, and descending modulatory responses to pain.

\section{ACKNOWLEDGMENTS}

This study was partly supported by a research grant from AstraZeneca AB, Mölndal, Sweden. 


\section{Authors' affiliations}

C H Wilder-Smith, D Schindler, Brain-Gut Research Group, Berne, Switzerland

K Lovblad, Department of Neuroradiology, University Hospital, Berne, Switzerland

S M Redmond, A Nirkko, Department of Neurology, University Hospital , Berne, Switzerland

\section{REFERENCES}

1 Drossman DA, Corazziari E, Talley NJ, et al. The functional gastrointestinal disorders. McLean: Degnon Associates, 2000:355-362.

2 Whitehead WE, Engel BT, Schuster MM. Irritable bowel syndrome: physiological and psychological differences between diarrhoea-predominant and constipation-predominant patients. Dig Dis Sci 1980;25:404-13.

3 Sun WM, Read NW, Prior A, et al. Sensory and motor responses to rectal distension vary according to rate and pattern of balloon inflation. Gastroenterology 1990;99:1008-15.

4 Hasler WL, Soudah HC, Owyang C. Somatostatin analog inhibits afferent response to rectal distension in diarrhoea-predominant irritable bowel patients. J Pharmacol Exp Ther 1994;268:1206-11

5 Hammer J, Phillips SF, Talley NJ, et al. Effect of a 5-HT3 antagonist (ondansetron) on rectal contractility and compliance in health and the irritable bowel syndrome. Aliment Pharmacol Ther 1993;7:543-51.

6 Lembo T, Munakata J, Mertz H, et al. Evidence for the hypersensitivity of the lumbar splanchnic afferents in irritable bowel syndrome. Gastroenterology 1994: 107:1686-96

7 Mertz H, Naliboff B, Munakata J, et al. Altered rectal perception is a biological marker of patients with irritable bowel syndrome. Gastroenterology 1995; 109:40-52.

8 Munakata J, Naliboff B, Harraf F, et al. Repetitive sigmoid stimulation induces rectal hyperalgesia in patients with irritable bowel syndrome. Gastroenterology 1997; 1 12:55-63.

9 Naliboff BD, Munakata J, Fullerton S, et al. Evidence for two distinct perceptual alterations in irritable bowel syndrome. Gut 1997;41:505-12.

10 Ritchie J. Pain from distension of the pelvic colon by inflating a balloon in the irritable colon syndrome. Gut 1973;14:125-32.

11 Chang L, Munakata J, Mayer EA, et al. Perceptual responses in patients with inflammatory and functional bowel disease. Gut 2000;47:497-505.

12 Schmulson M, Chang L, Naliboff B, et al. Correlation of symptom criteria with perception thresholds during rectosigmoid distension in irritable bowel syndrome patients. Am J Gastroenterol 2000;95:152-6.

13 Mayer EA, Gebhart GF. Basic and clinical aspects of visceral hyperalgesia. Gastroenterology 1994;107:271-93.

14 Willer JC, De Brouker T, Le Bars D. Encoding of nociceptive thermal stimuli by diffuse noxious inhibitory controls in humans. J Neurophysiol 1989;62:1028-38.

15 Willer JC, Bouhassira D, Le Bars D. Neurophysiological basis of the counterirritation phenomenon: diffuse control inhibitors induced by nociceptive stimulation. Neurophysiol Clin 1999;29:379-400.

16 Silverman DH, Munakata JA, Ennes $\mathrm{H}$, et al. Regional cerebral activation in normal and pathological perception of visceral pain. Gastroenterology 1997; 1 12:64-72.

17 Lautenbacher S, Rollman GB. Possible deficiencies of pain modulation in fibromyalgia. Clin J Pain 1997;13:189-95.

18 Kosek E, Hansson P. Modulatory influence on somatosensory perception from vibration and heterotopic noxious conditioning stimulation (HNCS) in fibromyalgia patients and healthy subjects. Pain 1997;70:41-51

19 Bouhassira D, Danziger H, Attal N, et al. Comparison of pain suppressive effects of clinical and experimental painful conditions. Brain 2003; 126:1068-78

20 Willer JC, Roby A, Le Bars D. Psychological and electrophysiological approaches to the pain-relieving effects of heterotopic nociceptive stimuli. Brain 1984; 107:1095-112.

21 Washington LL, Gibson SJ, Helme RD. Age-related differences in the endogenous analgesic response to repeated cold water immersion in human volunteers. Pain 2000;89:89-96.

22 Edwards RR, Fiilingim RB, Ness TJ. Age-related differences in endogenous pain modulation: a comparison of diffuse noxious inhibitory controls in pain modulation: a comparison of diffuse noxious inhibitory

23 Chadwick VS, Chen W, Shu D, et al. Activation of the mucosal immune system in irritable bowel syndrome. Gastroenterology 2002;122:1778-83.

24 Coffin B, Sabate J-M, Jian R, et al. Mechanisms of visceral pain in patients with inflammatory bowel disease or irritable bowel syndrome. 10th World Congress of Pain, 2002:1539-P139.

25 Mertz H, Morgan V, Tanner G, et al. Regional cerebral activation in irritable bowel syndrome and control subjects with painful and nonpainful rectal distention. Gastroenterology 2000;1 18:842-8.

26 Bonaz B, Baciu M, Papillon E, et al. Central processing of rectal pain in patients with irritable bowel syndrome: an fMRI study. Am J Gastroenterol 2002;97:654-61.

27 Thompson WG, Longstreth GF, Drossman DA, et al. Functional bowel disorders and functional abdominal pain. Gut 1999;45(suppl 2):1143-7.

28 Nirkko AC, Ozdoba C, Redmond SM, et al. Different ipsilateral representations for distal and proximal movements in the sensorimotor cortex: activation and deactivation patterns. Neuroimage 2001;13:825-35.

29 Nirkko AC. A small software utility for fully automated download and evaluation of FMRI data. Neurolmage 2000;11:S919.
30 Rademacher J, Galaburda AM, Kennedy DN, et al. Human cerebral cortex: localization, parcellation, and morphometry with magnetic resonance imaging. J Cogn Neurosci 1992;4:352-74.

31 Jaeggi SM, Seewer R, Nirkko AC. Does excessive memory load attenuate activation in the prefrontal cortex? Load-dependent processing in single and dual tasks: functional magnetic resonance imaging study. Neuroimage 2003; 19:210-25.

32 Verne GN, Himes NC, Robinson ME, et al. Central representation of visceral and cutaneous hypersensitivity in the irritable bowel syndrome. Pain 2003;103:99-110.

33 Fields HL, Basbaum Al. Central nervous system mechanism of pain modulation. In: Wall PD, Melzack R, eds. Textbook of pain, 4th edn. London: Churchill Livingstone, 1999:309-29.

34 Kakigi R. Diffuse noxious inhibitory controls. J Neurol Sci 1994;125:198-205.

35 Reinart A, Treede R, Bromm B. The pain inhibiting pain effect: an electrophysiological study in humans. Brain Res 2000;863:102-10.

36 Villanueva L, Le Bars D. The activation of bulbo-spinal controls by peripheral nociceptive inputs: diffuse noxious inhibitory controls. Biol Res 1995:28:113-25.

37 Bouhassira D, Villanueva L, Le Bars D. Effects of systemic morphine on diffuse noxious inhibitory controls: role of the periaquaductal grey. Eur J Pharmacol 1992;216:149-56.

38 Dubner R, Ren K. Endogenous mechanisms of sensory modulation. Pain 1999;(suppl 6):45-53.

39 Keay KA, Clement Cl, Owler B, et al. Convergence of deep somatic and visceral nociceptive information onto a discrete ventrolateral midbrain periaqueductal gray region. Neuroscience 1994;61:727-32.

40 Silverman DHS. Cerebral activity in the perception of visceral pain. Curr Rev Pain 1999:33:291-9.

41 Naliboff BD, Berman S, Chang L, et al. Sex-related differences in IBS patients: central processing of visceral stimuli. Gastroenterology 2003;124:1738-47.

42 Coghill RC, Talbot JD, Evans AC, et al. Distributed processing of pain and vibration by the human brain. J Neurosci 1994;14:4095-108.

43 Jones EG. Perception of visceral sensation. Advances in psychophysiology, vol 5. New York: Jessica Kingsley Publishers, 1994:55-192.

44 Casey KL, Minoshima S, Berger KL, et al. Positron emission tomographic analysis of cerebral structures activated specifically by repetitive noxious heat stimuli. J Neurophysiol 1994;71:802-7.

45 Talbot JD, Marrett S, Evans AC, et al. Multiple representations of pain in human cerebral cortex. Science 1991;251:1355-8.

46 Treede RD, Kenshalo DR, Gracely RH, et al. The cortical representation of pain. Pain 1999:79:105-11.

47 Derbyshire SW. Meta-analysis of thirty-four independent samples studied using PET reveals a significantly attenuated central response to noxious stimulation in clinical pain patients. Curr Rev Pain 1999;3:265-80.

48 Derbyshire SWG. Metareview of functional imaging studies with noxious stimuli. Gastroenterology 2001;120(suppl 1):3836.

49 Derbyshire SWG. Exploring the pain "neuromatrix". Curr Rev Pain 2000;6:467-77.

50 Pandya DN. Association cortex. In: Adelman G, Smith B, eds. Encyclopedia of neuroscience on CD-ROM, 2nd edn. Amsterdam: Elsevier Science, 2001

51 Serrien DJ, Nirkko AC, Loher TJ, et al. Movement control of manipulative tasks in patients with Gilles de la Tourette syndrome. Brain 2002;125:290-300.

52 Naliboff BD, Derbyshire SW, Munakata J, et al. Evidence for decreased activation of central fear circuits by expected aversive visceral stimuli in IBS patients. Gastroenterology 2000;1 18:A137.

53 Drevets WC, Price JL, Simpson JR, et al. Subgenual prefrontal cortex abnormalities in mood disorders. Nature 1997;386:824-7.

54 Petrovic $\mathbf{P}$, Ingvar M. Imaging cognitive modulation of pain processing. Pain 2002;95:1-5.

55 Buchel C, Bornhovd K, Quante M, et al. Dissociable neural responses related to pain intensity, stimulus intensity, and stimulus awareness within the anterior cingulate cortex: a parametric single-trial laser functional magnetic resonance imaging study. J Neurosci 2002;1:970-6.

56 Davidson RJ, Irwin W. The functional neuroanatomy of emotion and affective style. Trends Cogn Sci 1999;3:11-21.

57 Coderre TJ, Vaccarino, AL, Melzack R. Central nervous system plasticity in the tonic pain response to subcutaneous formalin injection. Brain Res 1990;535:155-18.

58 LeDoux JE. Emotional circuits in the brain. In: Adelman G, Smith B, eds. Encyclopedia of neuroscience on CD-ROM, 2nd edn. Amsterdam: Elsevier Science, 2001.

59 Mayer EA. The neurobiology of stress and gastrointestinal disease. Gut 2000;47:861-9.

60 Ploghaus A, Narain C, Beckmann CF, et al. Exacerbation of pain by anxiety is associated with activity in a hippocampal network. J Neurosci 2001;21:9896-903

61 Sawamoto N, Honda M, Okada T, et al. Expectation of pain enhances responses to nonpainful somatosensory stimulation in the anterior cingulate cortex and parietal operculum/posterior insula: an event-related functional magnetic resonance imaging study. J Neurosci 2000;20:7438-45.

62 Stehberg J, Acuna-Goycolea C, Ceric F, et al. The visceral sector of the thalamic reticular nucleus in the rat. Neuroscience 2001; 106:745-55.

63 Westlund KN. Visceral nociception. Curr Rev Pain 2000;4:478-87.

64 Al-Chaer ED, Westlund KN, Willis WD. Nucleus gracilis: an integrator for visceral and somatic information. J Neurophysiol 1997;78:521-7.

65 Swanson WL. The limbic system. In: Adelman G, Smith B, eds. Encyclopedia of neuroscience on CD-ROM, 2nd edn. Amsterdam: Elsevier Science, 2001

66 Wilder-Smith CH. Different sensory defects in IBS patients with constipation and diarrhoea? Gut 2003;52(suppl VI):A90. 